\title{
STUDY OF THREE ELASTIC-PLASTIC CONSTITUTIVE MODELS BY NON-PROPORTIONAL FINITE DEFORMATIONS OF OFHC COPPER
}

\author{
A. S. Khan and P. Cheng* \\ Department of Mechanical Engineering, University of Maryland, Baltimore, County, Baltimore, MD 21228, \\ U.S.A.
}

(Received in final revised form 3 March 1996)

\begin{abstract}
Experimental stress-strain data of OFHC copper first under torsion to $13 \%$ and then under torsion-tension to about $10 \%$ are used to study the characteristics of three elastic-plastic constitutive models: Chaboche's super-positional nonlinear model, Dafalias and Popov's two surface model and Watanabe and Atluri's version of the endochronic model. The three models, originally oriented for infinitesimal deformation, have been extended for finite deformation. The results show (a) the Misestype yield surface used in the three models brings about significant departure of the predictions from the experimental data; (b) Chaboche's and Dafalias' models are easier than Watanabe and Atluri's model in determining the material parameters in them, and (c) Chaboche's and Watanabe \& Atluri's models produce almost the same prediction to the data, while Dafalias' model cannot accurately predict the plastic deformations when a loading path changes in its direction. Copyright (C) 1996 Elsevier Science Ltd
\end{abstract}

\section{INTRODUCTION}

After extensive investigations of both theoretical considerations and experiments, it is recognized that constitutive models based on the Mises-type yield surface with kinematical and isotropic hardening is a good approximation for the description of time-independent behaviour of elastic-plastic materials under infinitesimal and stable deformations. Consequently, many researchers in this area devoted their efforts to establishing the relationship between kinematic hardening and plastic deformation. These efforts resulted in many constitutive models. Among the commonly used models in recent literature are Prager's linear model [1949], Armstrong and Frederick's nonlinear model [1966], Chaboche [1986], Mroz's multi-surface model [1967], Dafalias and Popov's twosurface model [1976], Valanis [1971] endochronic model and its development by Watanabe and Atluri [1986].

In face of many constitutive models, one may wonder how to choose a model for a specific problem. Each model was based on some experimental facts and did not fully discuss other situations. It seems interesting to evaluate some of the commonly used models, especially considering their merits and limitations for some typical experimental

*Permanent address: Institute of Mechanics, Chinese Academy of Sciences, Beijing 100080, Peoples' Republic of China. 
data. In fact, a few researchers have made efforts in this respect. McDowell [1987] experimentally evaluated the non-proportional cyclic behavior of Mroz's, Dafalias and Popov's, Chaboche's and Tseng-Lee's models. Inoue et al. $[1989,1991]$ gave a comprehensive comparison between their experiments and the predictions of 10 models, including those by Chaboche, Mroz and Valanis, on plasticity, creep and their interaction.

This paper evaluates the three elastic-plastic constitutive models: Chaboche's superpositional nonlinear model [1986], Dafalias and Popov's two surface model [1976] and Watanabe and Atluri's version of endochronic model [1986]. Emphasis is placed on the suitability of the three models to a set of experimental stress-strain data, given by Khan and Wang [1993], of OFHC copper first under tension to around $13 \%$ and then under torsion and tension to about $10 \%$. In Section II, the experimental procedure is briefly presented. Section III gives the description of the deformation and stress of a thin-walled tube under finite deformation and correlates the measured stresses and deformations based on a general constitutive relationship. In Section IV, the equations of the three constitutive models are provided in a systematic way. These models were originally proposed for the case of infinitesimal deformation. In order to predict a deformation of about $20 \%$, these models are extended to finite deformation by changing the rates of stress and kinematic hardening from the material rates for infinitesimal deformation to the corotated objective rates with elastic spin for finite formation. The determination of the parameters in the three models is given in Section V. The experimental data and the predictions of the three models are presented and discussed in the fifth and sixth sections.

\section{EXPERIMENTAL PROCEDURES}

The material used in this study was OFHC copper. Thin-walled tubular specimens, with a test section approximately $1.2 \mathrm{in}$. $(30.48 \mathrm{~mm})$ long, having an inside diameter of $0.375 \mathrm{in}$. $(9.53 \mathrm{~mm})$ and an outside diameter of $0.45 \mathrm{in} .(11.43 \mathrm{~mm})$, were used for all the experiments presented. Before the tests all the specimens were annealed in an oxygen-free atmosphere at $1100^{\circ} \mathrm{F}\left(593.3^{\circ} \mathrm{C}\right)$ for an hour and then cooled in a furnace to room temperature.

The testing machine was a dead weight type capable of combined tension-torsion loadings. Water flow with a constant flow rate was used as the loadings in order to maintain constant normal stress or shear stress rate. Details of the machine were described by Khan and Parikh [1986].

The loading paths are depicted by Fig. 1, in which path $\mathrm{O} \rightarrow \mathrm{C}$ was the first stage torsion to a plastic state, path $\mathrm{C} \rightarrow U$ was the torsion which unloaded partially the specimen and the paths beginning at point $U$ with angles $0^{\circ}, 15^{\circ}, 30^{\circ}, 45^{\circ}, 60^{\circ}, 75^{\circ}, 90^{\circ}$ and $180^{\circ}$ to the shear stress axis were the second stage nonproportional loadings.

The deformation during the first stage torsion and the partial unloading is recorded by a TRANS-TEK angular displacement transducer. During the combined tension and torsion, strain gauges are used to measure the deformations in the directions of the tube axis and $\pm 45^{\circ}$ angles to the tube axis. Details of the measurement were reported in the paper by Khan and Wang [1993].

\section{DEFORMATION AND STRESS OF THIN-WALLED TUBE}

Before we consider the material behavior of the specimen, we need to generally derive its deformation and stress in order to clarify the relationship between the measured stresses and the measured deformations under finite deformation. 


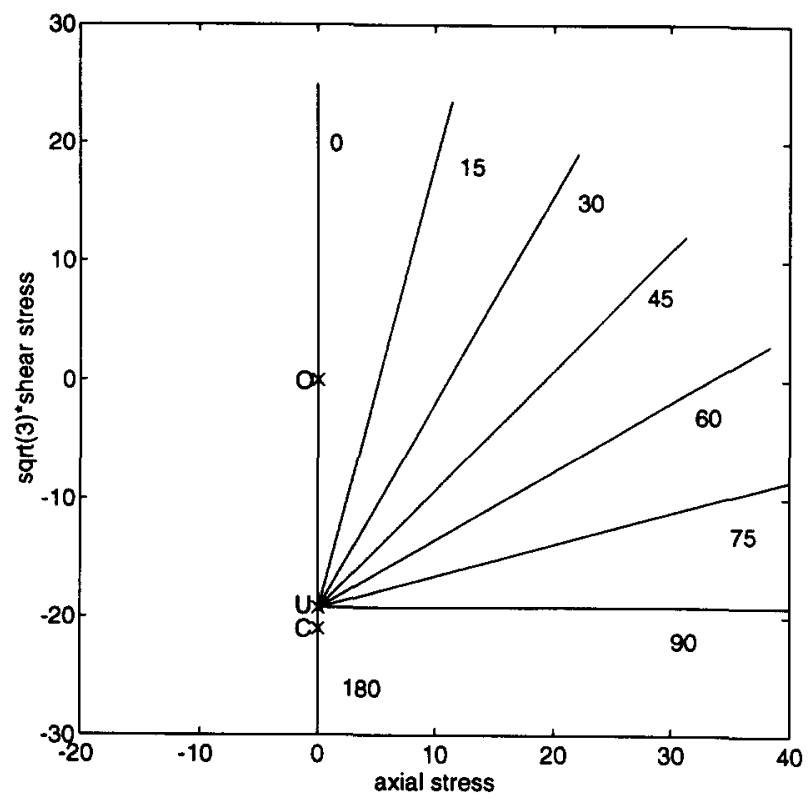

Fig. 1. Loading paths in the $\sigma-\sqrt{3} \tau$ space.

Let $L, R_{1}$ and $R_{2}$ denote the initial length, inner and outer radii of the tube, respectively. Since the tube is expected to deform under the loading in tension and torsion, the deformation can be described as

$$
\begin{aligned}
& r=\alpha R \\
& \theta=\Theta+\omega Z \\
& z=\lambda Z
\end{aligned}
$$

where $(r, \theta, z)$ and $(R, \Theta, Z)$ are the positions a point after and before the deformation in a cylindrical coordinate system. The origin locates the center of one end of the tube and the $Z$-axis coincides with the tube axis, $\omega$ is the twisting angle per unit length and $\alpha$ and $\lambda$ are the ratios of radii and lengths before and after deformation.

From the above deformation, the deformation and velocity gradients, $\mathbf{F}$ and $\mathbf{L}$, can be found (e.g. Khan \& Huang [1995]):

$$
\mathbf{F}=\alpha \mathbf{e}_{r} \otimes \mathbf{e}_{R}+\alpha \mathbf{e}_{\theta} \otimes \mathbf{e}_{\Theta}+\omega \alpha R_{m} \mathbf{e}_{\theta} \otimes \mathbf{e}_{Z}+\lambda \mathbf{e}_{z} \otimes \mathbf{e}_{Z}
$$

and

$$
\begin{aligned}
\mathbf{L}=\dot{\mathbf{F}} \cdot \mathbf{F}^{-1} & =\frac{\dot{\alpha}}{\alpha} \mathbf{e}_{r} \otimes \mathbf{e}_{r}-\dot{\omega} Z \mathbf{e}_{r} \otimes \mathbf{e}_{\theta}+\dot{\omega} Z \mathbf{e}_{\theta} \otimes \mathbf{e}_{r} \\
& +\frac{\dot{\alpha}}{\alpha} \mathbf{e}_{\theta} \otimes \mathbf{e}_{\theta}+\dot{\phi} \mathbf{e}_{\theta} \otimes \mathbf{e}_{z}+\frac{\dot{\lambda}}{\lambda} \mathbf{e}_{z} \otimes \mathbf{e}_{z}
\end{aligned}
$$


in which ( $\left.\mathbf{e}_{R}, \mathbf{e}_{\Theta}, \mathbf{e}_{Z}\right)$ and $\left(\mathbf{e}_{r}, \mathbf{e}_{\theta}, \mathbf{e}_{z}\right)$ are the base vectors of a point in the tube before and after deformation, $\phi$ is defined by

$$
\dot{\phi}=\frac{\alpha R_{m} \dot{\omega}}{\lambda}
$$

and $R_{m}=\left(R_{1}+R_{2}\right) / 2$ has been used to replace $R$ in a thin-walled tube.

Accordingly, the strain rate and material spin of the specimen are

$$
\begin{aligned}
\mathbf{D} & =\frac{1}{2}\left(\mathbf{L}+\mathbf{L}^{T}\right)=\frac{\dot{\alpha}}{\alpha} \mathbf{e}_{r} \otimes \mathbf{e}_{r}+\frac{\dot{\alpha}}{\alpha} \mathbf{e}_{\theta} \otimes \mathbf{e}_{\theta} \\
& +\frac{\dot{\phi}}{2} \mathbf{e}_{\theta} \otimes \mathbf{e}_{z}+\frac{\dot{\phi}}{2} \mathbf{e}_{z} \otimes \mathbf{e}_{\theta}+\frac{\dot{\lambda}}{\lambda} \mathbf{e}_{z} \otimes \mathbf{e}_{z}
\end{aligned}
$$

and

$$
\begin{aligned}
\mathbf{W} & =\frac{1}{2}\left(\mathbf{L}-\mathbf{L}^{T}\right)=-\dot{\omega} Z \mathbf{e}_{r} \otimes \mathbf{e}_{\theta}+\dot{\omega} Z \mathbf{e}_{\theta} \otimes \mathbf{e}_{r} \\
& +\frac{\dot{\phi}}{2} \mathbf{e}_{\theta} \otimes \mathbf{e}_{z}-\frac{\dot{\phi}}{2} \mathbf{e}_{z} \otimes \mathbf{e}_{\theta} .
\end{aligned}
$$

Now, let $P$ and $M$ denote the axial tensile force and the twisting moment. Due to the loading symmetry, the stress in the tube will be

$$
\boldsymbol{\sigma}=\sigma_{\theta \theta} \mathbf{e}_{\theta} \otimes \mathbf{e}_{\theta}+\sigma_{z \theta} \mathbf{e}_{z} \otimes \mathbf{e}_{\theta}+\sigma_{\theta z} \mathbf{e}_{\theta} \otimes \mathbf{e}_{z}+\sigma_{z z} \mathbf{e}_{z} \otimes \mathbf{e}_{z},
$$

where

$$
\sigma_{z z}=\frac{P}{2 \pi r\left(R_{2}-R_{1}\right)}=\frac{P}{2 \pi \alpha R_{m}\left(R_{2}-R_{1}\right)}
$$

and

$$
\sigma_{z \theta}=\frac{M}{2 \pi r^{2}\left(R_{2}-R_{1}\right)}=\frac{M}{2 \pi \alpha^{2} R_{m}^{2}\left(R_{2}-R_{1}\right)}
$$

This stress and the above material spin produce the following Jaumann rate of the stress:

$$
\begin{aligned}
\breve{\boldsymbol{\sigma}} & =\dot{\boldsymbol{\sigma}}-\mathbf{W} \cdot \boldsymbol{\sigma}+\boldsymbol{\sigma} \cdot \mathbf{W} \\
& =\left(\dot{\sigma}_{\theta \theta}-\dot{\phi} \sigma_{\theta z}\right) \mathbf{e}_{\theta} \otimes \mathbf{e}_{\theta}+\left(\dot{\sigma}_{\theta z}+\left(\sigma_{\theta \theta}-\sigma_{z z}\right) \frac{\dot{\phi}}{2}\right) \mathbf{e}_{\theta} \otimes \mathbf{e}_{z} \\
& +\left(\dot{\sigma}_{\theta z}+\left(\sigma_{\theta \theta}-\sigma_{z z}\right) \frac{\dot{\phi}}{2}\right) \mathbf{e}_{z} \otimes \mathbf{e}_{\theta}+\left(\dot{\sigma}_{z z}+\dot{\phi} \sigma_{\theta z}\right) \mathbf{e}_{z} \otimes \mathbf{e}_{z} .
\end{aligned}
$$


Suppose that the constitutive relation of the specimen is described by

$$
\hat{\boldsymbol{\sigma}}=C: D
$$

where $\mathbf{C}$ is the elastic-plastic modulus, then by substituting the strain and stress rates, eqns (5) and (10) into this constitutive relation, six equations will be obtained. Among these six equations the two equations which correspond to the stress components $\sigma_{r \theta}$ and $\sigma_{r z}$ are expected to be naturally satisfied because of the symmetrical deformation. The remaining four equations are used to determine the four unknown variables $\dot{\alpha}, \dot{\lambda}, \dot{\omega}$ and $\dot{\sigma}_{\theta \theta}$ if the loading rates $\dot{P}$ and $\dot{M}$ are prescribed, or alternatively to determine the four unknown variables $\dot{P}, \dot{M}, \dot{\alpha}$ and $\dot{\sigma}_{\theta \theta}$ if the deformation rates $\dot{\lambda}$ and $\dot{\omega}$ are provided. In this way, a loading history will be related to a corresponding deformation history, if the constitutive relation is specified.

\section{THREE CONSTITUTIVE MODELS FOR FINITE ELASTIC-PLASTIC DEFORMATION}

In the phenomenological theory of finite plasticity for engineering metals, the following assumptions are usually adopted:

(1) Velocity gradient $\mathbf{L}$ is decomposed into elastic part $\mathbf{L}^{e}$ and plastic part $\mathbf{L}^{p}$ as

$$
\mathbf{L}=\mathbf{L}^{e}+\mathbf{L}^{p}
$$

and each part is sub-decomposed into strain rate and spin:

$$
\mathbf{D}^{e}=\frac{1}{2}\left(\mathbf{L}^{e}+\mathbf{L}^{e T}\right), \quad \mathbf{W}^{e}=\frac{1}{2}\left(\mathbf{L}^{e}-\mathbf{L}^{e T}\right)
$$

and

$$
\mathbf{D}^{p}=\frac{1}{2}\left(\mathbf{L}^{p}+\mathbf{L}^{p T}\right), \quad \mathbf{W}^{p}=\frac{1}{2}\left(\mathbf{L}^{p}-\mathbf{L}^{p T}\right)
$$

where the superscript " $T$ " means transpose.

(2) Elastic strain rate follows Hooke's law:

$$
\breve{\boldsymbol{\sigma}}=\dot{\boldsymbol{\sigma}}-\mathbf{W}^{e} \cdot \boldsymbol{\sigma}+\boldsymbol{\sigma} \cdot \mathbf{W}^{e}=\mathbf{C}^{e}: \mathbf{D}^{e}
$$

where $\dot{\sigma}$ is the material rate of stress and

$$
\mathbf{C}^{e}=2 G \mathbf{I}_{4}+\frac{2 G \nu}{1-2 \nu} \mathbf{I} \otimes \mathbf{I}
$$

is the elastic modulus in which $G$ is the shear modulus, $\nu$ is Poisson's ratio, $\mathbf{I}$ and $\mathbf{I}_{\mathbf{4}}$ are the unit second- and fourth-order tensors.

(3) Plastic deformation occurs when the following Mises-type yield criterion is satisfied with kinematic and isotropic hardening:

$$
\sqrt{\frac{3}{2}(\mathbf{s}-\alpha):(\mathbf{s}-\alpha)}-\sigma_{y}-r=0
$$


where $\sigma_{y}$ is initial tensile yield stress, $\alpha$ is kinematic hardening, $r$ is isotropic hardening and $\mathbf{s}=\boldsymbol{\sigma}-\operatorname{tr}(\boldsymbol{\sigma}) \mathbf{I} / 3$ is deviatoric stress. Especially for continuing plastic deformation, the yield criterion gives the following consistency condition:

$$
\mathbf{N}: \dot{\mathbf{s}}=\mathbf{N}: \dot{\alpha}+\sqrt{\frac{2}{3}} \dot{r}
$$

where $\dot{\alpha}$ and $\dot{r}$ are the material rates of $\alpha$ and $r$ and

$$
\mathbf{N}=\frac{\mathbf{s}-\boldsymbol{\alpha}}{\sqrt{(\mathbf{s}-\boldsymbol{\alpha}):(\mathbf{s}-\boldsymbol{\alpha})}}
$$

(4) Plastic strain rate obeys the normality rule

$$
\mathbf{D}^{p}=p \mathbf{N}
$$

where $p$ is called the magnitude of plastic strain rate.

(5) The rates of isotropic and kinematic hardening are proportional to the magnitude of plastic strain rate:

$$
\dot{r}=E_{r} p
$$

and

$$
\breve{\alpha}=\dot{\alpha}-\mathbf{W}^{e} \cdot \alpha+\alpha \cdot \mathbf{W}^{e}=\mathbf{E}_{\alpha} p
$$

where $E_{r}$ and $\mathbf{E}_{\alpha}$ are the moduli relating the hardening to the plastic deformation.

(6) Plastic spin is proportional to the magnitude of plastic strain rate:

$$
\mathbf{W}^{p}=\mathbf{M} p
$$

where $\mathbf{M}$ is an anti-symmetrical tensor factor.

From these assumptions, the elastic-plastic modulus $\mathbf{C}$ in eqn (11) can be found to be

$$
\mathbf{C}=\mathbf{C}^{e}-\frac{2 G}{2 G+E_{p}}(2 G \mathbf{N}+\mathbf{M} \cdot \boldsymbol{\sigma}-\boldsymbol{\sigma} \cdot \mathbf{M}): \mathbf{N}
$$

where

$$
E_{p}=\mathbf{N}: \mathbf{E}_{\alpha}+\sqrt{\frac{2}{3}} E_{r}=\frac{\mathbf{N}: \breve{\mathbf{\sigma}}}{p}
$$

is known as plastic modulus. Therefore, if these assumptions are used for the formulation, then the task of developing a constitutive model turns to be that of specifying $E_{r}, \mathbf{E}_{\alpha}$ and $M$, i.e. the evolution of isotropic and kinematic hardening and plastic spin.

The evolution of isotropic hardening can be directly determined by one-dimensional. tensile or torsional, stress-strain experimental data. Several experimental studies show that the following assumption is a good approximation:

$$
\dot{r}=\beta\left(R_{m}-r\right) p
$$


where $R_{m}$ and $\beta$ are two material parameters. In fact, this assumption states that isotropic hardening is an exponential function of plastic deformation with maximum value $R_{m}$ and shape-controlled parameter $\beta$. In terms of the modulus of isotropic hardening,

$$
E_{r}=\beta\left(R_{m}-r\right)
$$

As for the relation between plastic spin and plastic deformation the Dafalias suggestion [1983] is widely accepted:

$$
\mathbf{W}^{p}=\eta\left(\boldsymbol{\alpha} \cdot \mathbf{D}^{p}-\mathbf{D}^{p} \cdot \boldsymbol{\alpha}\right)=\eta p(\boldsymbol{\alpha} \cdot \mathbf{N}-\mathbf{N} \cdot \boldsymbol{\alpha})
$$

where $\eta$ is a function of plastic deformation. In our opinion, plastic spin normally has the same order as the plastic strain rate. Therefore, it is proposed that

$$
\eta=\frac{1}{\sqrt{(\boldsymbol{\alpha} \cdot \mathbf{N}-\mathbf{N} \cdot \boldsymbol{\alpha}):(\boldsymbol{\alpha} \cdot \mathbf{N}-\mathbf{N} \cdot \boldsymbol{\alpha})}}
$$

With these considerations, the modulus of plastic spin becomes

$$
\mathbf{M}=\frac{\boldsymbol{\alpha} \cdot \mathbf{N}-\mathbf{N} \cdot \boldsymbol{\alpha}}{\sqrt{(\boldsymbol{\alpha} \cdot \mathbf{N}-\mathbf{N} \cdot \boldsymbol{\alpha}):(\boldsymbol{\alpha} \cdot \mathbf{N}-\mathbf{N} \cdot \boldsymbol{\alpha})}}
$$

The evolution of kinematic hardening has been one of key issues in the phenomenological theory of elastic-plastic constitutive relation. Many competitive models for it have been proposed. In the following section, three models which are often used in recent literature are represented. These models were originally proposed for use with infinitesimal deformation. Here, these models are extended to finite deformation by changing their rate of kinematic hardening from the material rate for infinitesimal deformation to the corotational rate with elastic spin. That is, $\breve{\alpha}=\dot{\alpha}-W^{e} \cdot \boldsymbol{\alpha}+\boldsymbol{\alpha} \cdot \mathbf{W}^{e}$ is used to replace $\dot{\alpha}$ in these original models.

\section{IV.1. Chaboche's super-positional nonlinear model}

Chaboche [1981] proposed that kinematic hardening might be composed of say, $n$ parts, as

$$
\alpha=\sum_{i=1}^{n} \boldsymbol{\alpha}^{(i)}
$$

and that each part evolves with plastic deformation as in Armstrong and Frederick's [1966] exponential function:

$$
\breve{\boldsymbol{\alpha}}^{(i)}=\beta_{c}^{(i)}\left(\alpha_{c}^{(i)} \mathbf{D}^{p}-\boldsymbol{\alpha}^{(i)} p\right)
$$

where $\beta_{c}^{(i)}$ and $\alpha_{c}^{(i)}$ are material parameters, representing the saturated values and shapecontrolled parameters. 
By using eqn (20), eqns (31) and (32) yield the modulus of kinematic hardening:

$$
E_{\alpha}=\sum_{i}^{n} \beta_{c}^{(i)}\left(\alpha_{c}^{(i)} N-\alpha^{(i)}\right) .
$$

\section{IV.2. Endochronic model and its modification by Watanabe and Atluri}

Contrary to the usually used rate form of an elastic-plastic constitutive model, Valanis [1971] proposed an integral form:

$$
s=\int_{-\infty}^{z} \rho\left(z-z^{\prime}\right) \frac{\partial \mathbf{\epsilon}^{p}}{\partial z^{\prime}} \mathrm{d} z^{\prime}
$$

where $\boldsymbol{\epsilon}^{p}$ is plastic strain (for infinitesimal deformation $\dot{\boldsymbol{\epsilon}}^{p}=\mathbf{D}^{p}$ ), $z$ is an intrinsic time which is defined by

$$
\mathrm{d} z=\frac{p}{f\left(\int p\right)}
$$

and $\rho$ and $f$ are two material functions. But Watanabe and Atluri [1986] found that if the material function $\rho$ was assumed to be

$$
\rho(z)=\frac{2}{3} \rho_{0} \delta(z)+\sum_{i=1}^{n} \rho^{(i)} \mathrm{e}^{-h^{(i)} z}
$$

where $\delta(z)$ is the Dirac function of $z, \rho_{0}, h^{i}$ and $\rho^{i}$ are material parameters, then eqn (34) was equivalent to (a) the Mises-type yield criterion with isotropic and kinematic hardening of eqn (17), (b) the normality rule eqn (20) and (c) the following evolution of kinematic hardening:

$$
\alpha=\sum_{i=1}^{n} \alpha^{(i)}
$$

and

$$
\breve{\boldsymbol{\alpha}}^{(i)}=\beta_{v}^{(i)}\left(\alpha_{v}^{(i)} \mathbf{N}-\frac{\sigma_{y}+R_{m}}{\sigma_{y}+r} \boldsymbol{\alpha}^{(i)} p\right)
$$

where

$$
\begin{gathered}
\alpha_{v}^{(i)}=\frac{R_{m}+\sigma_{y}}{\rho_{0} h^{(i)}} \rho^{(i)} \\
\beta_{v}^{(i)}=\frac{\rho_{0} h^{(i)}}{R_{m}+\sigma_{y}}
\end{gathered}
$$


and

$$
r=\rho_{0} f\left(\int p\right)-\sigma_{y}
$$

Obviously, this model gives the modulus of kinematic hardening as

$$
E_{\alpha}=\sum_{i}^{n} \gamma^{(i)}\left(\alpha_{v}^{(i)} \mathbf{N}-\frac{\sigma_{y}+R_{m}}{\sigma_{y}+r} \boldsymbol{\alpha}^{(i)}\right)
$$

\section{IV.3. Dafalias and Popov's two surface model}

In order to describe the evolution of kinematic hardening, Dafalias and Popov [1975] introduced another criterion called bounding surface. They proposed (a) that the bounding surface might have the Mises-type form:

$$
\sqrt{\frac{3}{2}\left(\mathbf{s}_{b}-\alpha_{b}\right):\left(\mathbf{s}_{b}-\alpha_{b}\right)}-\sigma_{0 b}-r_{b}=0
$$

where $\sigma_{0 \mathrm{~b}}, \boldsymbol{\alpha}_{b}$ and $r_{\mathrm{b}}$ are similarly called initial yield stress, kinematic and isotropic hardening of bounding surface and $s_{b}$ is the deviatoric stress at bounding surface; (b) $\mathbf{s}_{b}$ was determined by making the normal of the bounding surface at this point identical to that of yield surface at the loading points $\mathbf{s}$, i.e.

$$
\mathbf{s}_{b}=\boldsymbol{\alpha}_{b}+\frac{\sigma_{0 b}+r_{b}}{\sigma_{0}+r}(\mathbf{s}-\boldsymbol{\alpha})
$$

(c) the kinematic hardening of the yield and bounding surfaces evolve with plastic deformation in the direction from the loading stress to the stress at the bounding surface:

$$
\breve{\boldsymbol{\alpha}}=\dot{\mu} \frac{\mathbf{s}_{b}-\mathbf{s}}{\delta}, \quad \breve{\boldsymbol{\alpha}}_{b}=\dot{\mu}_{b} \frac{\mathbf{s}_{b}-\mathbf{s}}{\delta}
$$

where $\delta=\sqrt{\left(\mathbf{s}_{b}-\mathbf{s}\right):\left(\mathbf{s}_{b}-\mathbf{s}\right)}, \dot{\mu}$ and $\dot{\mu}_{b}$ are two factors to be determined and (d) the plastic modulus $E_{p}$ depends on the distance $\delta$ from the loading point to the stress point at bounding surface such that

$$
E_{p}=E_{p b}+h\left(\delta_{0}\right) \frac{\delta}{\delta_{0}-\delta}
$$

where $\delta_{0}$ is the initial value of $\delta$ at the beginning of continuous plastic deformation, $h\left(\delta_{0}\right)$ is a material function of $\delta_{0}$ and $E_{b p}$ is defined by

$$
E_{p b}=\frac{1}{p} \mathbf{N}: \breve{\mathbf{s}}_{b}=\frac{1}{p} \mathbf{N}:\left(\dot{\mathbf{s}}_{b}-\mathbf{W}^{e} \cdot \mathbf{s}_{b}+\mathbf{s}_{b} \cdot \mathbf{W}^{e}\right) .
$$


By substituting eqn (45) for $\breve{\alpha}$ into eqns (22) and (25), factor $\dot{\mu}$ is found to be

$$
\dot{\mu}=p\left(E_{p}-\sqrt{\frac{2}{3}} E_{r}\right) \frac{\delta}{\mathbf{N}:\left(\mathbf{s}_{b}-\mathbf{s}\right)} .
$$

Similarly, if we let

$$
E_{r b}=\frac{\dot{r}_{b}}{p}
$$

denote the modulus of the isotropic hardening of the bounding surface, the factor $\dot{\mu}_{b}$ can be found to be

$$
\dot{\mu}_{b}=p\left(E_{p b}-\sqrt{\frac{2}{3}} E_{r b}\right) \frac{\delta}{\mathbf{N}:\left(\mathbf{s}_{b}-\mathbf{s}\right)}
$$

Dafalias and Popov [1976] described $E_{r b}$ as a material parameter and once $E_{r b}$ is specified, the two kinematic hardening, one for yield surface and the other for the bounding surface, are formulated.

\section{DETERMINATION OF MATERIAL PARAMETERS}

In the preceding constitutive models, two parameters, $G$ and $\nu$, are included for the description of elastic behavior. To describe plastic behavior, the initial yield stress $\sigma_{y}$, the saturated value of isotropic hardening, $R_{m}$ and shape-controlled parameter $\beta$ are involved. More parameters are included for the description of kinematic hardening. In Chaboche's model, if kinematic hardening is composed of two parts, i.e. $n=2$ in eqn (31) as Chaboche [1986] suggested, the parameters will be $\alpha_{c}^{(1)}, \alpha_{c}^{(2)}, \beta_{c}^{(1)}$ and $\beta_{c}^{(2)}$. Similarly, four parameters, $\alpha_{v}^{(1)}, \alpha_{v}^{(2)}, \beta_{v}^{(1)}$ and $\beta_{v}^{(2)}$ appear in Watanabe and Atluri's model, if $n=2$ is chosen for eqn (37). Dafalias and Popov's model includes parameter $\sigma_{y b}$ and two functions $h\left(\delta_{0}\right)$ and $E_{r b}$. All of these material parameters and functions are the characteristics of a specific material and need to be determined by experiments.

Figure 2 shows the experimental data in solid lines of engineering shear stress-strain of fully-annealed OFHC copper. Section OC is the first stage torsion. Section CU is the unloading before the non-proportional loadings are applied, section UF is the reversal loading which is shown in Fig. 1 with path $0^{\circ}$ and section UCD is the reloading which is shown in Fig. 1 with $180^{\circ}$. In order to determine the parameters for this material, points $A$ and $E$ are labeled on this curve. The two points are the initial and reversal yield points which are determined by amplifying the sections $O A$ and $C U$ and using $0.2 \%$ offset strain as the yield criterion. The values of points $A, C, D, E$ and $F$ are listed in Table 1.

From the data at points $A, C$ and $E$, the elastic shear modulus $G$ is found by averaging the slops of sections $O A$ and $C E$. The initial yield stress $\sigma_{y}=\sqrt{3}(1.732)=3$ ksi. Poisson' ratio cannot be determined by this curve, but is estimated to be $1 / 3$. 
Determining the other parameters from the data in Table 1 is not straightforward. When a tubular specimen is deformed by a twisting moment, the specimen undergoes not only a torsional deformation, but also an extension. The coupling of the two types of deformation makes it difficult, if not impossible, to find an explicit relation between the twisting moment and the torsional deformation from a general constitutive equation, such as any of the above three models. As a result, the data in Table 1 cannot be related to the other parameters in an explicit form. In practice, a numerical curve fitting method is usually used to solve the problem. In this paper in order to reduce the complexity of finding the parameters for the material, we divide the parameter-determining procedure into two steps. The first step is to get estimated values of the parameters by simplifying the problem and using infinitesimal deformation. For infinitesimal deformation, the coupling does not exist and an explicit relationship between the twisting moment and the torsional deformation can be obtained from Chaboche's and Dafalias and Popov's models. Thus, it is possible to use the data in Table 1 to directly determine the parameters. Since the maximum deformation in this set of experiments is about $20 \%$, the estimated values of the parameters are expected to be a good approximation of their actual values. The second

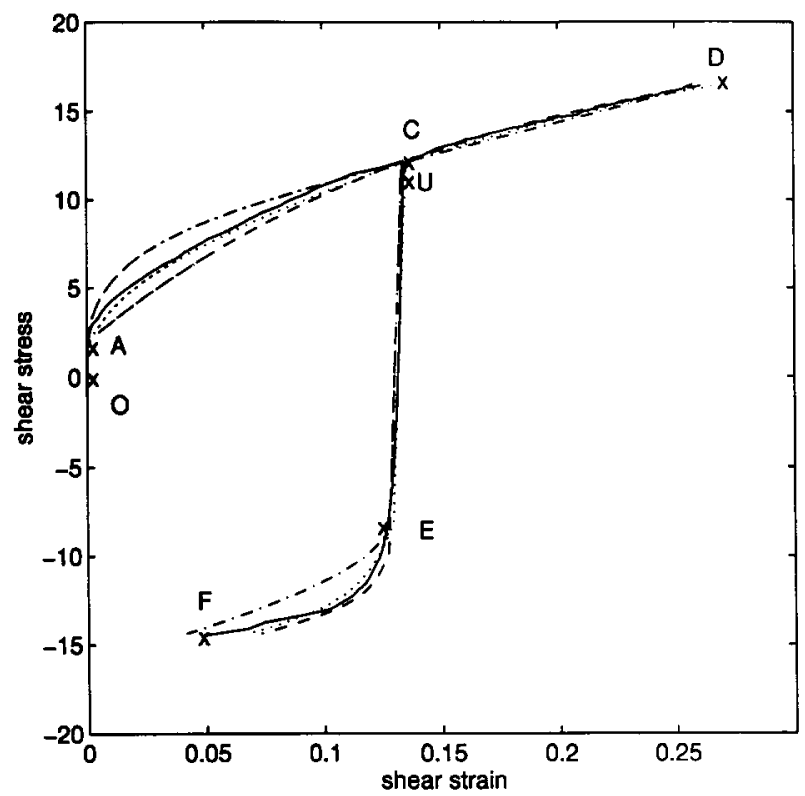

Fig. 2. Comparison of shear stress-strain relation under torsion deformation — experiments, - - Endochronic model, - . - . - Dafalias' model, . . . Chaboche's model.

Table 1. Some data of Fig. 2

\begin{tabular}{lllllr}
\hline Points & A & C & D & E & F \\
\hline Shear strain $\gamma$ & 0.0005 & 0.134 & 0.269 & 0.128 & 0.042 \\
Plastic shear strain $\gamma^{p}$ & 0.0 & 0.132 & 0.264 & 0.132 & 0.046 \\
Shear stress $\tau$ ksi & 1.732 & 12.2 & 16.7 & -8.3 & -14.5 \\
\hline
\end{tabular}


step is to modify the estimated values of the parameters in the numerical implementation of the three models to values such that they make the theoretical prediction of the models match the whole set of experimental data as closely as possible.

Under infinitesimal deformation, the stress and strain rate of the specimen under the twisting moment have only nontrivial components of $\sigma_{\theta z}$ and $D_{\theta z}$ and so do the stress rate, plastic stain rate and kinematic hardening. Also, the stress and all the rates involved can be replaced by the engineering stress and material rates. Therefore, let $\gamma^{p}=2 D_{\theta z}^{p}, \alpha=\alpha_{\theta z}$ and $\tau=\sigma_{\theta z}$, then $p= \pm \dot{\gamma}^{p}$ where ' + ' is for $\dot{\gamma}^{p}>0$ and ' $\cdots$ ' is for $\dot{\gamma}^{p}<0$, and the yield surface of eqn (17) becomes

$$
\tau=\alpha \pm \frac{\sigma_{y}+r}{\sqrt{3}}
$$

Since in the three models, $\alpha$ and $\mathrm{r}$ are supposed to depend on plastic deformation, the hardening at points $A, C$ and $E$ can be found from the data in Table 1:

$$
r_{A}=0, \quad r_{C}=r_{E}=\sqrt{3}\left(\frac{12.2+8.3}{2}-\sqrt{3}\right)=14.75
$$

and

$$
\alpha_{A}=0, \quad \alpha_{C}=\alpha_{E}=\frac{12.2-8.3}{2}=1.95
$$

Meanwhile, let $r_{0}, \alpha_{0}, \alpha_{0}^{(i)}$ and $\gamma_{0}^{p}$ denote the initial values of $r, \alpha, \alpha^{(i)}$ and $\gamma^{p}$, respectively. Then, the evolution equation of isotropic hardening and the Chaboche kinematic hardening formulations, eqns (26), (31) and (32), can be integrated into

$$
r-r_{0}=\left(R_{m}-r_{0}\right)\left(1-\mathrm{e}^{\mp \beta\left(\gamma^{p}-\gamma_{0}^{p}\right)}\right)
$$

and

$$
\alpha-\alpha_{0}=\sum_{i=1}^{2}\left(\alpha^{(i)}-\alpha_{0}^{(i)}\right)
$$

where

$$
\alpha^{(i)}-\alpha_{0}^{(i)}=\frac{1}{2}\left( \pm \alpha_{c}^{(i)}-\alpha_{o}^{(i)}\right)\left(1-\mathrm{e}^{\mp \beta_{c}^{(i)}\left(\gamma^{p}-\gamma_{0}^{p}\right)}\right)
$$

In order to determine the parameters included in the above equations, the plastic shear strains, $\gamma^{p}=\gamma-\tau / G$, at the points $A, C, D, E$ and $F$ are calculated and presented in Table 1. Thus, for the loading process $A \mathrm{C}$, eqns (54)-(56) are

$$
R_{m}\left(1-\mathrm{e}^{-0.132 \beta}\right)=14.75
$$


and

$$
\sum_{i=1}^{2} \alpha_{C}^{(i)}=1.95
$$

where

$$
\alpha_{C}^{(i)}=\frac{1}{2} \alpha_{c}^{(i)}\left(1-\mathrm{e}^{-0.132 \beta_{c}^{(i)}}\right)
$$

Similarly, the application of eqns (54)-(56) as well as eqn (51) to the loading process $C D$ and the reversal loading process $E F$ yields

$$
\begin{gathered}
r_{D}=R_{m}\left(1-\mathrm{e}^{-0.264 \beta}\right) \\
r_{F}=r_{E}+\left(R_{m}-r_{E}\right)\left(1-\mathrm{e}^{-0.086 \beta}\right)
\end{gathered}
$$

and

$$
\begin{aligned}
& \sum_{i=1}^{2} \alpha_{D}^{(i)}=16.7-\frac{3+r_{D}}{\sqrt{3}} \\
& \sum_{i=1}^{2} \alpha_{F}^{(i)}=-14.5+\frac{3+r_{F}}{\sqrt{3}}
\end{aligned}
$$

where

$$
\begin{gathered}
\alpha_{D}^{(i)}=\frac{1}{2} \alpha_{c}^{(i)}\left(1-\mathrm{e}^{-0.264 \beta_{c}^{(i)}}\right) \\
\alpha_{F}^{(i)}=\alpha_{E}-\frac{1}{2}\left(\alpha_{c}^{(i)}+\alpha_{E}\right)\left(1-\mathrm{e}^{-0.086 \beta_{c}^{(i)}}\right)
\end{gathered}
$$

Equations (57)-(65) cannot give a unique solution for the parameters in them. But if the isotropic and kinematic hardening are expected to be monotonically increased with plastic deformation, i.e. $\beta>0, \beta_{c}^{(i)}>0$ and $\alpha_{c}^{(i)}>0$ are required, the equations provide the following estimation: $\beta=5.30, R_{m}=29.30, \alpha_{c}^{(1)}=3.20, \alpha_{c}^{(2)}=1.37, \beta_{c}^{(1)}=12$ and $\beta_{c}^{(2)}$ $=32$.

The above procedure can not be applied to estimate the parameters in the Watanabe and Atluri model, since the evolution equation of the kinematic hardening can not be integrated into such a difference form as eqns (55) and (56) when the exponential function of isotropic hardening is used. In the numerical implementation of the model, we 
use $\beta=5.30, R_{m},=29.30, \alpha_{0}^{(1)}=3.20, \alpha_{v}^{(2)}=1.37, \beta_{v}^{(1)}=12$ and $\beta_{v}^{(2)}=32$, the same estimated values as those in Chaboche's model, since the parameters in the two models are the same in number and very similar in their physical interpretations.

In order to estimate the parameters in Dafalias and Popov's model, we use the curves in Fig. 2 to further specify the bounding surface. By assuming that the bounding surface is composed of the two parallel asymptotic lines of the curves through points $D$ and $F$, it is found that the stress values on the bounding surface at the points which correspond to the loading point $C$ and the reversal loading point $E$ are almost the same. That suggests that there is almost no kinematic hardening for the bounding surface. Therefore, it is assumed that the kinematic hardening of the bounding surface is zero and the isotropic hardening is a linear function of plastic deformation, i.e. $\alpha_{b}=0$ and $\dot{r}=\sqrt{3 / 2} E_{p b} p$ where $E_{p b}$ is a constant. From Fig. 2, $E_{p b}$ and the initial yield stress at the bounding surface $\sigma_{y b}$ are found to be 47.60 and 13.53 , respectively. Further, in the case of infinitesimal shear deformation, $\delta= \pm \sqrt{2}\left(\tau_{b}-\tau\right)$, and eqns (25) and (47) reduce to $\sqrt{2} \dot{\tau}=E_{p} \dot{\gamma}^{p}$ and $\sqrt{2} \dot{\mathrm{r}}_{b}$ $=E_{p b} \dot{\gamma}^{p}$. Thus, by using eqn (46),

$$
\dot{\delta}= \pm\left(E_{p b} \dot{\gamma}^{p}-\sqrt{2} \dot{\tau}\right)=\mp h\left(\delta_{0}\right) \frac{\delta}{\delta_{0}-\delta} \dot{\gamma}^{p}
$$

or written in a different form,

$$
\delta-\delta_{0}= \pm\left(E_{p b}\left(\gamma^{p}-\gamma_{0}^{p}\right)-\sqrt{2}\left(\tau-\tau_{0}\right)\right)
$$

and

$$
h\left(\delta_{0}\right)=\mp \frac{1}{\gamma^{p}-\gamma_{0}^{p}}\left(\delta-\delta_{0}-\delta_{0} \ln \frac{\delta}{\delta_{0}}\right) .
$$

For the loading process $A C$,

$$
\delta_{A}=\sqrt{\frac{2}{3}}\left(\sigma_{y b}-\sigma_{y}\right)=\sqrt{\frac{2}{3}}(13.53-3)=8.60
$$

and eqns (67) and (68) give

$$
\delta_{C}=8.60+47.60 * 0.132-\sqrt{2}(12.2-\sqrt{3})=0.08
$$

and

$$
h(8.60)=\frac{1}{0.132}\left(0.08-8.60-8.60 \ln \frac{0.08}{8.60}\right)=240.20
$$

Similarly, for the loading process $C D$ and the reversal loading process $E F$, it is found that $\delta_{D}=0.0001, \delta_{E}=5.59, \delta_{F}=0.92, h(0.08)=3.45$ and $h(5.59)=62.98$. Therefore. if $h\left(\delta_{0}\right)$ is supposed to be a polynomial of degree two, the obtained three values of $h$ will yield $h\left(\delta_{0}\right)=5.11-21.19 \delta_{0}+5.64 \delta_{0}^{2}$.

Beginning with the estimated values, the parameters are modified and determined through the numerical implementation of the three models with the aim of finding the best fit between each model and the experimental data. The final values of the parameters are listed in Table 2. 


\section{EXPERIMENTAL DATA AND PREDICTIONS OF THE THREE MODELS}

Figure 2 shows the experimental data and the three models' predictions of simple shearing deformation. It is found that the three models predict the experimental data well. This is understandable and reasonable because the data at four points $C, D, E$ and $F$ have been used to estimate the material parameters in the models. From this figure the numerical analysis is inferred to be accurate enough.

Figures 3--5 give the comparisons of experimental strains with the predictions of the three models for the torsion-tension loadings in different directions. It is found that the predicted directions of the plastic strain rate depart significantly from the experimental data.

Figures 6-8 are the results of shear stress-strain relations. It is shown that the three models preclict the experiments well.

Figures 9-11 show the axial stress-strain relations. It is found that experimental data are usually higher than the predicted curves of the three models.

Table 2. Material parameters in the models

\begin{tabular}{|c|c|c|}
\hline Chaboche model & Watanabe-Atluri model & Dafalias-Popov model \\
\hline$R_{m}=31$ & $R_{m}=25$ & \\
\hline$\beta=4.8$ & $\beta=6.4$ & $R_{m}=31$ \\
\hline$\alpha_{c}^{(1)}=2.2$ & $\alpha_{v}^{(1)}=5$ & $\beta=4.8$ \\
\hline$\alpha_{c}^{(2)}=1.7$ & $\alpha_{v}^{(2)}=1.7$ & $E_{p b}=47.60$ \\
\hline$\beta_{c}^{(1)}=115$ & $\beta_{v}^{(1)}=115$ & $h=5.11-21.19 \delta_{0}+5.64 \delta_{0}^{2}$ \\
\hline$\beta_{c}^{(2)}=60$ & $\beta_{v}^{(2)}=60$ & \\
\hline
\end{tabular}

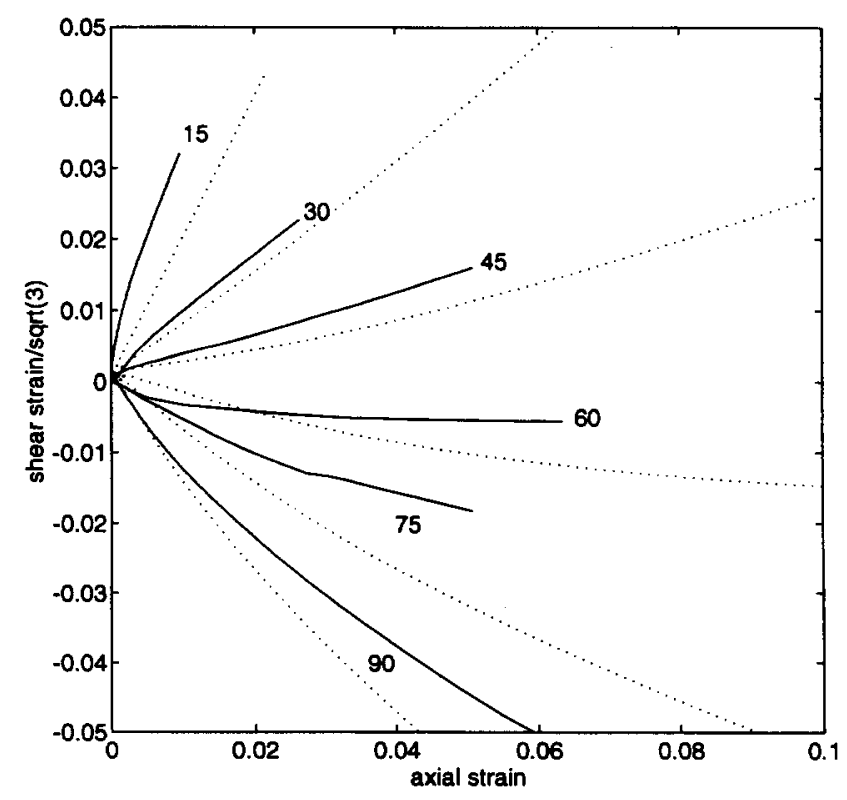

Fig. 3. Comparison of directions of plastic strain rates — experiments, ---- Chaboche's model. 


\section{DISCUSSION}

VII.1. Isotropic and kinematic hardening

The experimental data in Fig. 2 shows that after $13.4 \%$ shear deformation the annealed copper has a higher level of isotropic hardening as compared with its kinematic hard-

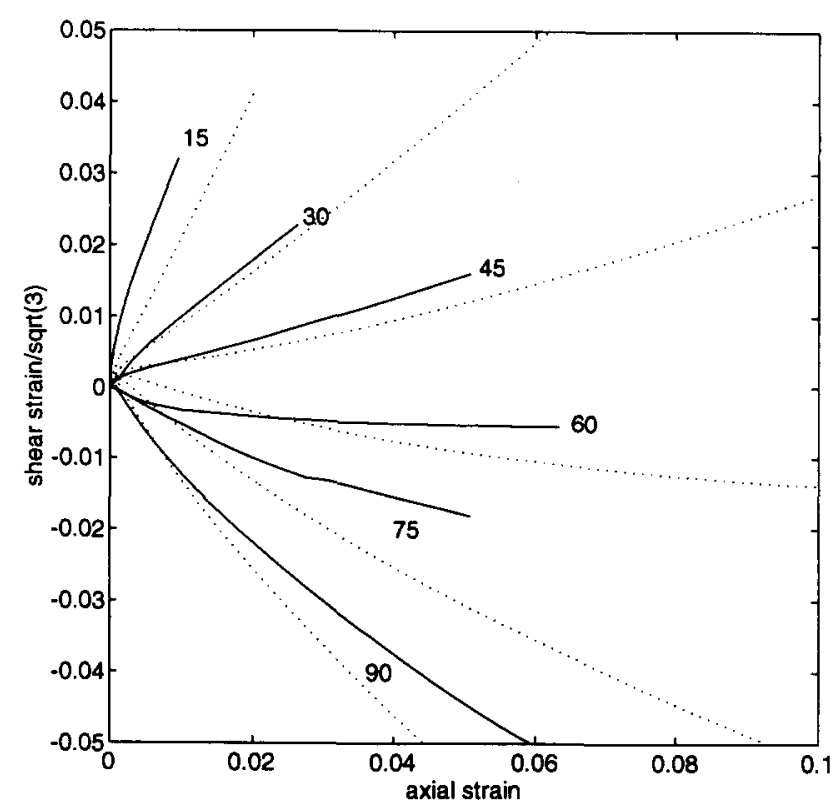

Fig. 4. Comparison of directions of plastic strain rates _- experiments, - - - Endochronic model.

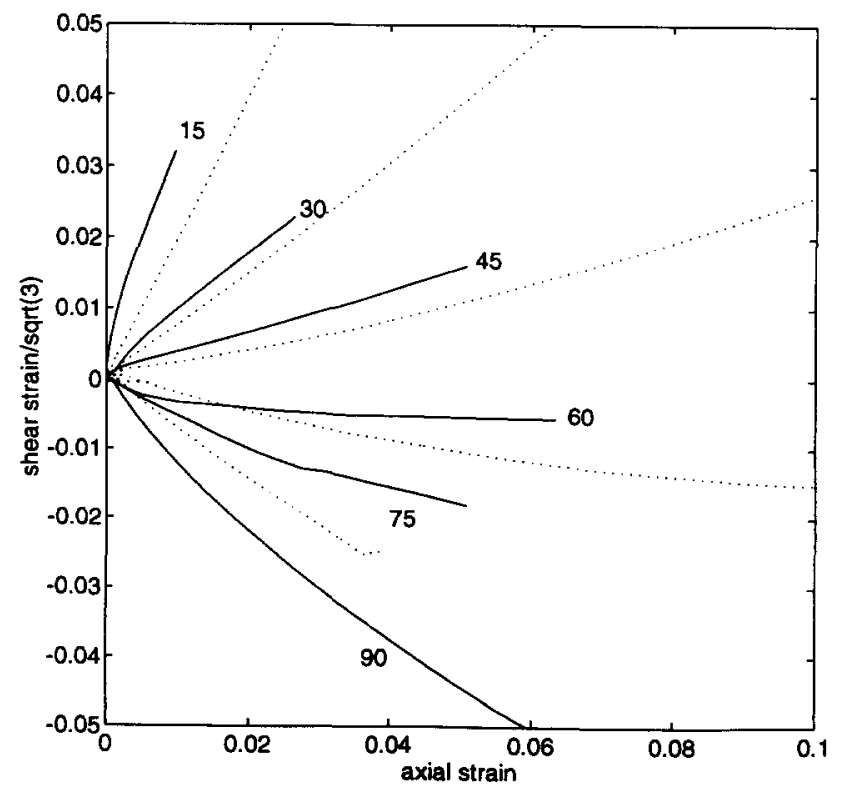

Fig. 5. Comparison of directions of plastic strain rates __ experiments, $-\ldots+$ Dafalias' Model. 
ening. The ratio of the two hardening is about $4: 1$. Accordingly, in Table 2 the shapecontrolled parameter for the isotropic hardening is much smaller than the parameters for the kinematic hardening. This means that at the beginning of plastic deformation, the increasing rate of isotropic hardening is smaller than that of kinematic hardening.

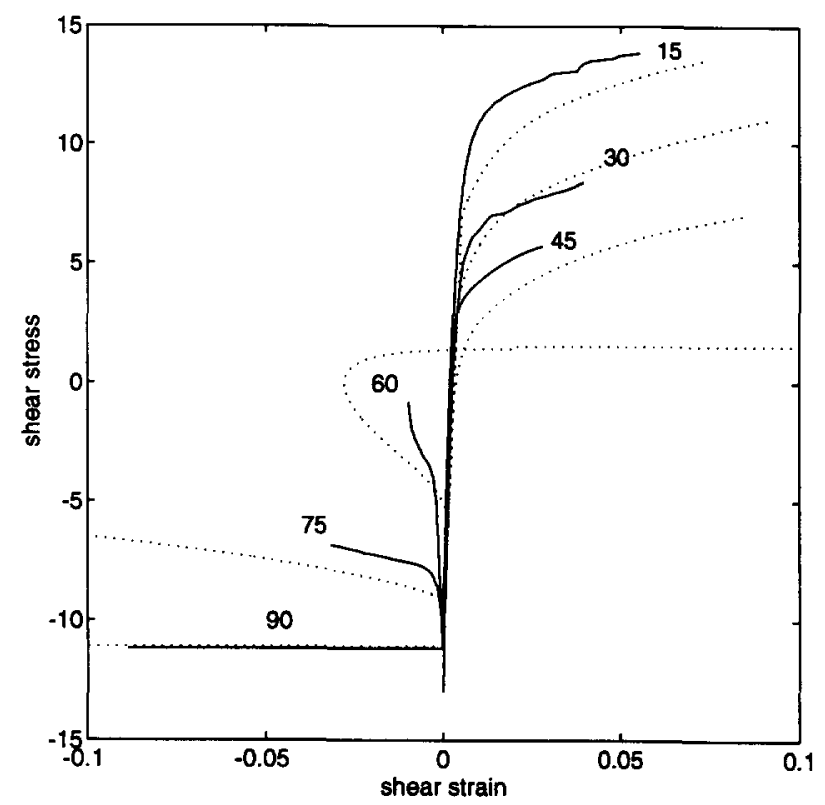

Fig. 6. Comparison of shear stress-strain relations under tension-torsion deformation experiments, - - - Chaboche's model.

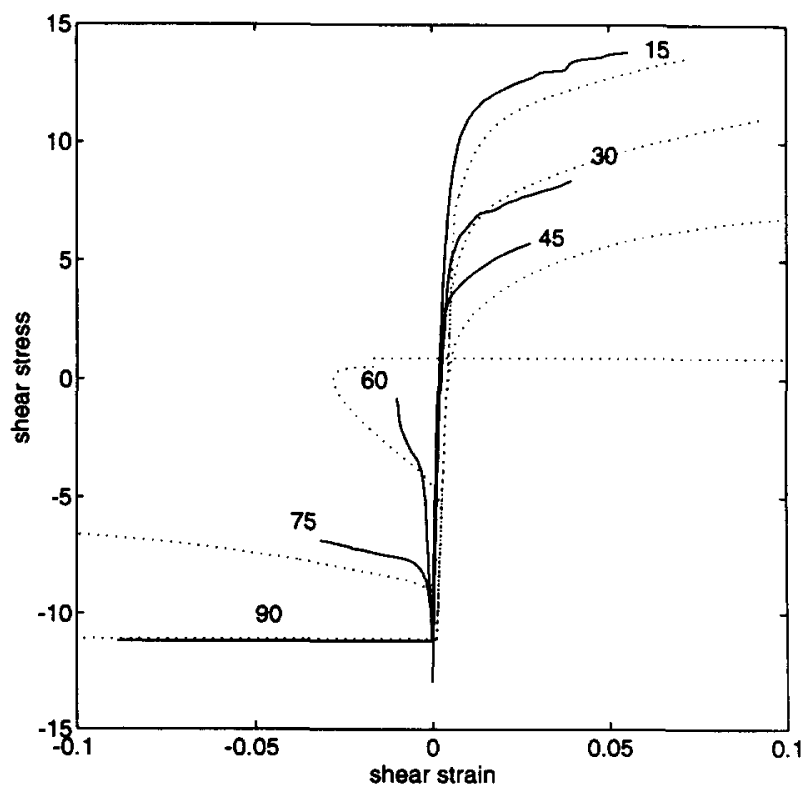

Fig. 7. Comparison of shear stress-strain relations under tension-torsion deformation - experiments, - _ - Endochronic model. 
Chaboche's model provides an example. When plastic shear strain is $1 \%$, the ratio of the isotropic hardening to the kinematic hardening is about 0.66 . This phenomenon, the changed dominant hardening from kinematic to isotropic, has also been observed in experiments on other materials. Phillips and Das [1985] concentrated their study on small

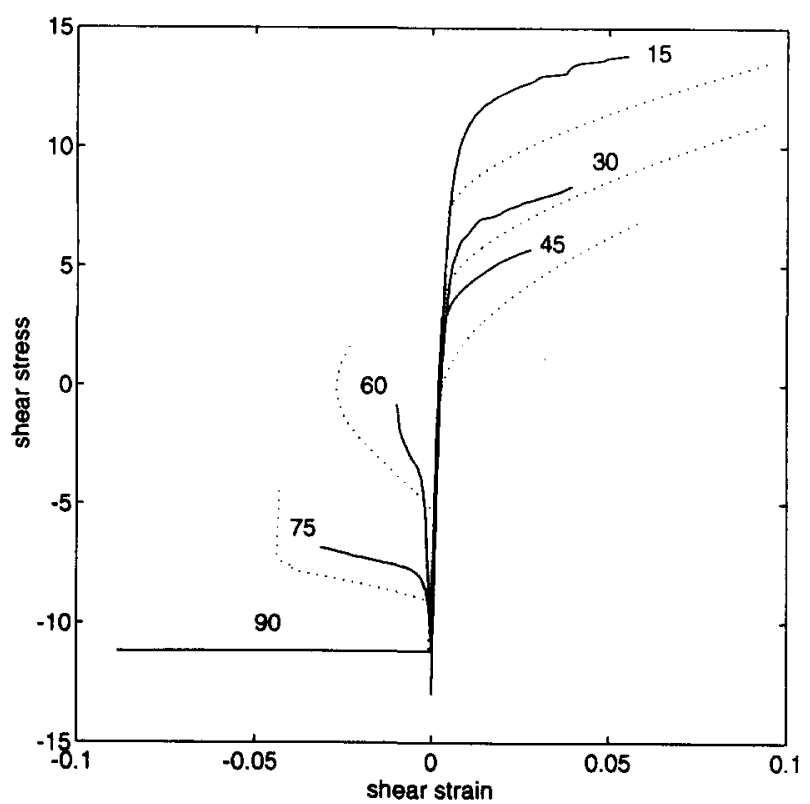

Fig. 8. Comparison of shear stress-strain relations under tension-torsion deformation - - - Dafalias' model.

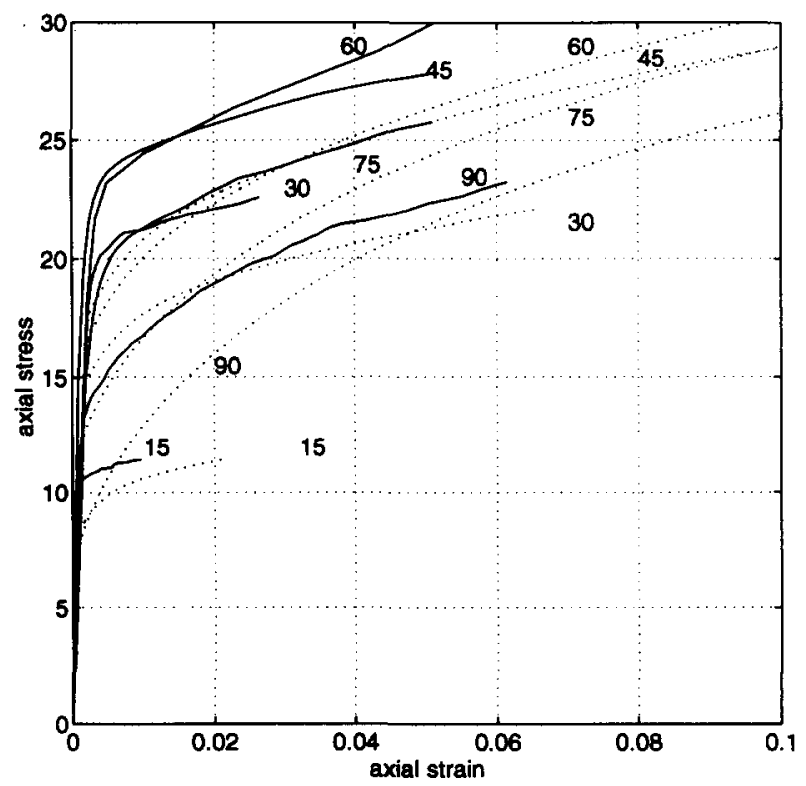

Fig. 9. Comparison of tensile stress-strain relations under tension-torsion deformation - experiments, --- Chaboche's model. 
deformation and found that the subsequent yield surfaces of commercially pure aluminum and brass move significantly in their position but change less in their sizes in the direction perpendicular to the load. Stout et al. [1985] investigated the evolution of the yield surface of aluminum 1100 with deformations from 3 to $250 \%$ and found that yield surfaces

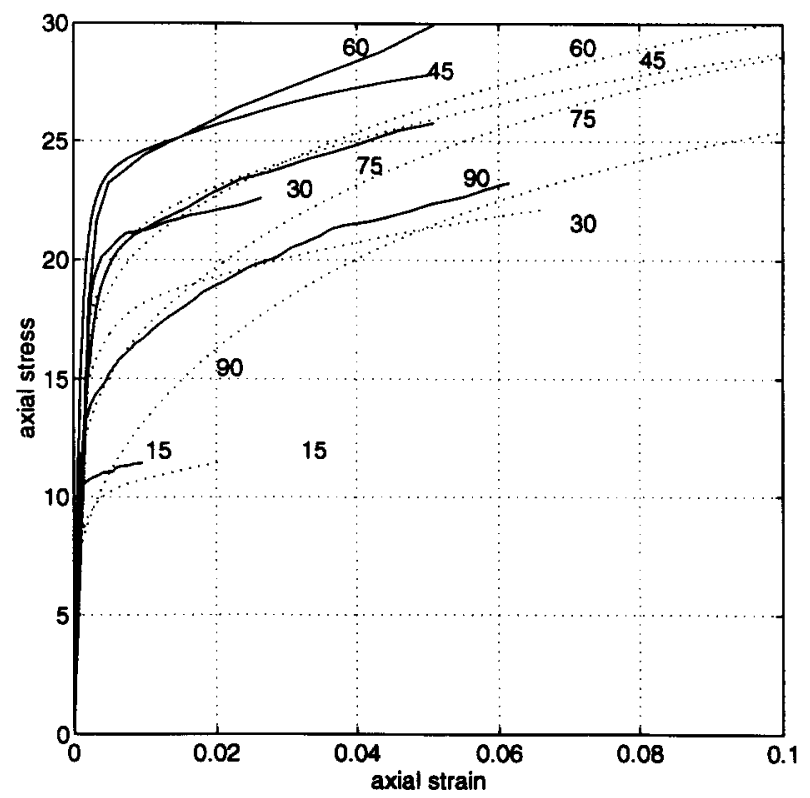

Fig. 10. Comparison of tensile stress-strain relations under tension-torsion deformation experiments, - - - Endochronic model.

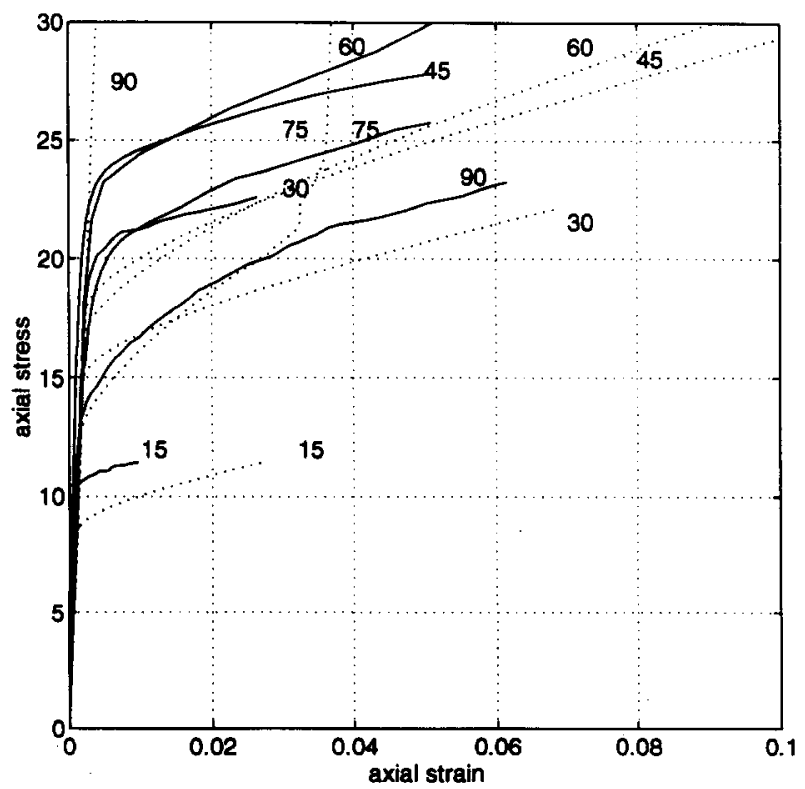

Fig. 11. Comparison of tensile stress-strain relations under tension-torsion deformation experiments, - - - Dafalias' model. 
change in size. This phenomenon seems counter to the expectation that kinematic hardening is related to texture development and should become larger when a specimen is increasingly deformed in one direction. Stout et al. [1985] suggested that this phenomenon may result from different micromechanisms corresponding to the magnitude of deformation.

\section{VII.2. The shape of the yield surface}

The yield surface changes, not only in its position and size but also in its shape, with the development of plastic deformation. Figure 12 shows the experimental data on the $0.2 \%$ offset yield surface after the specimen was deformed in shearing to $13.4 \%$ (the dash line is the connected experimental data). This result is found to be in accordance with the those by Phillips and Das [1985] and by Stout et al. [1985]. At the torsional preloading point, a sharply rounded corner develops while in the region opposite to the preloading point, the subsequent yield surface was flattened. The smaller the offset strain, the more the distortion of the subsequent yield surface.

In the three models, the position and size of yield surface are represented by kinematic hardening and isotropic hardening and no variable is introduced to correspond to the shape distortion of the yield surface. The assumption used in the three models results in a departure of the predictions from the experimental data, as shown in Figs 3-11. In the numerical prediction, the subsequent yield surface is the Mises-type circle and passes through the shear loading and reversal reloading points, as shown by the dash line in Fig. 12. Comparison of the normals of the dash and solid lines reveals that the experimental data have larger angles to the tensile axis than do the theoretical values. Consequently, the experimentally measured ratios of shear and tensile plastic strain rates are generally deviated positively from the theoretical results of the three models as shown in Figs 3-5. Meanwhile, since the distorted yield surface takes its smallest length in the preloading

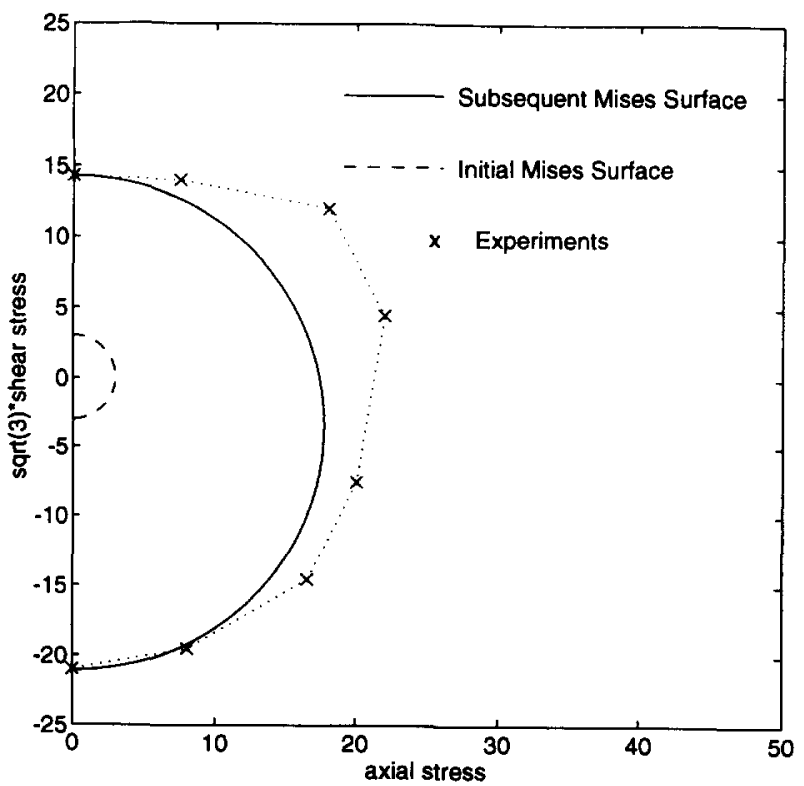

Fig. 12. Comparison of subsequent yield surface after torsion deformation. 
direction, the elastic region included by the Mises-type circle is smaller than that obtained by experiment. That is why, in Figs 6-11, the predicted stress-strain curves are generally underneath the experimental data.

From the numerical point of view, it is possible to make some of the predictions closer to the experimental data by using a different definition of yielding. For example, increasing the offset strain will yield a higher isotropic hardening and move the prediction upward toward the experimental data. But numerical results show that it is difficult to make all of the predictions match the experimental data. For example, increasing the offset strain makes the predicted stress-strain curves in Figs 6-11 closer to experimental data, but worsens the predicted results of simple shearing stress-strain relation in Fig. 2 and the directions of plastic strain rate in Figs 3-5. On the other hand, if the offset strain is adopted to be very small, as is expected in theory, the results in Fig. 2 and Figs 6-11 will be worse and those in Figs 3-5 will be better.

The Mises-type yield surface with kinematic and isotropic hardenings is a good approximation to experimental data for small deformations as considered in most published papers. But for moderate deformation, e.g. $10 \%$, the yield surface undergoes a significant distortion and the studied specimen will show strong anisotropic behavior. Only kinematic hardening may not suffice to describe the anisotropy. This difficulty opens an important issue of extending the three models to include the strong anisotropic behavior induced by a moderate plastic deformation.

\section{VII.3. Applications of the three models}

Figures 2-11 show that the three models are all equally capable of predicting the experimental data and it is difficult to designate one model superior to another except that for some problem due to changing loading directions in case of the two-surface model. The ideas for formulating the models are simple and plain that they can be easily understood and applied by engineers.

The three models have almost the same number of parameters, which makes them equally easy to apply. In addition to the two parameters for describing isotropic hardening, four parameters describe kinematic hardening in Chaboche's superpositional nonlinear model and in Watanabe and Atluris endochronic model. Five kinematic hardening parameters are used in Dafalias and Popov's two-surface model.

However, each of the three models has its individual features in determining material pararmeters. Chaboche's model and Dafalias and Popov's model can provide an explicit form of stress-strain relations in simple cases such as simple tension, compression and shearing, so that it is easy to determine or give equations for parameters as we did in the third section. However, the parameters in the endochronic model must be determined by examining the predicted results and iterating. In this aspect Chaboche's model and the two-surface model seem to be more convenient for application engineering.

In the determination of parameters for the super-positional nonlinear model and the two-surface model, Section III shows that some parameters are sensitive to the experimental values, but others are not, e.g. the maximum kinematic hardening and the asymptotic plastic modulus. The sensitivity of parameters to experimental data comes from the exponential and logarithmic functions which correspond to the short transient region from the beginning of plastic deformation to stable plastic deformation. This sensitivity 
may produce an unreasonable situation that for the same model and the same experimental data, different methods may produce different parameters.

For the two-surface model, there are some possibilities to improve the results. One of them, as Dafalias and Popov [1976] suggested in their paper, is to add kinematic hardening to the bounding surface. Another possibility is to avoid eqn (45), the Mroz method of prescribing the evolution of kinematic hardenings of yield and bounding surfaces. The two-surface model provides more flexibility in prediction, but realizing the model's possibilities is difficult, since improving the accuracy means increasing the complexity. Dafalias and Popov's [1976] suggestion is not acceptable for dealing with the loading paths with sharp changes in direction, i.e. when $\delta \leq \delta_{0}, \delta=\delta_{0}$ for the paths of $75^{\circ}$ and $90^{\circ}$. This is another open problem for the two-surface model.

\section{CONCLUSIONS}

In order to evaluate some typical constitutive models of elasto-plastic material, a set of stress-strain experimental data for fully annealed copper first under torsion to $13 \%$ and then under torsion-tension to $10 \%$ are presented. The data show that the $13 \%$ shear deformation produces a great deal of isotropic hardening while kinematic hardening is relatively small. This trend requires more phenomenological experiments and micromechanical analysis, since it cannot be explained by dislocation-based mechanisms of texture developments.

Chaboche's superpositional nonlinear model, Watanabe and Atluri's endochronic model and Dafalias and Popov's two-surface model are evaluated by comparing their predictions with the experimental data. It is found that the three models provide fair to good quality predictions of the experimental data except that the two-surface model has difficulty with the loading paths with sharp changes of directions.

There are two major modifications that need to be addressed in the three models in order to make their predictions agree better with the experimental data. One is to take the shape distortion of the yield surface into consideration. The other is to specify a detailed procedure for determining their parameters.

Acknowledgments - The financial support of the National Science Foundation, through grant No. MSS-9296045, Mechanics and Materials Program, is sincerely appreciated.

\section{REFERENCES}

1949 Prager, W., "Recent Developments in the Mathematic Theory of Plasticity," J. Appl. Phys., 20235.

1966 Armstrong, P.J. and Frederick, C.O., "A Mathematical Representation of the Multiaxial Bauschinger Effect," G.E.G.B. Report RD/B/N 731.

1967 Mroz, Z., "On the Description of Anisotropic Work-Hardening," J. Mech. Phys. Solids, $15,163$.

1971 Valanis, K.C., "A Theory of Viscoplasticity Without a Yield Surface, Part I: General Theory," Arch. Mech, 23517.

1971 Valanis, K.C., "A Theory of Viscoplasticity Without a Yield Surface, Part II: Application to the Mechanical Behavior of Metals," Arch. Mech., 23, 535.

1975 Michno, M.J. and Findley, W.N., "Subsequent Yield Surface for Annealed Mild Steel under ServoControlled Strain and Load Histories: Aging, Normality, Convexity, Corners, Bauschinger and Cross Effects," J. Engng Mater. Technol., 25, 25.

1976 Dafalias, Y.F. and Popov, E.P., "Plastic Internal Variables Formulatism of Cyclic Plasticity," ASME J. Appl. Mech., 43, 645.

1982 Valanis, K.C. and Lee, C.F., "Some Recent Developments of the Endochronic Theory with Application," Nuclear Engng Design, 69, 327. 
1983 Dafalias, Y.F., "Corotational Rates for Kinematic Hardening at Large Plastic Deformations," ASME J. Appl. Mech., 50, 561.

1984 Valanis, K.C. and Lee, C.F., "Endochronic Theory of Cyclic Plasticity with Applications," J. Appl. Mech., 51, 367.

1985 Phillips, A. and Das, P. K., "Yield Surfaces and Loading Surfaces of Aluminium and Brass: An Experimental Investigation at Room and Elevated Temperatures," Int. J. Plasticity, 1, 89.

1985 Stout, M. G., Martin, P. L., Helling, D. E. and Canova, G. R., "Multiaxial Yield Behavior of 1100 Aluminium Following Various Magnitudes of Prestrain,” Int. J. Plasticity, 1, 163.

1986 Chaboche, J.L., "Time-Independent Constitutive Theories for Cyclic Plasticity," Int. J. Plasticity, 2, 149.

1986 Khan, A.S. and Parikh, Y., "Large Deformation in Polycrystalline Copper under Combined TensionTorsion Loading, Unloading and Reloading or Reverse Loading: a Study of Two Incremental Theories of Plasticity," Int. J. Plasticity, 2, 379.

1986 Ohno, N. and Kachi, Y., "A Constitutive Model of Cyclic Plasticity for Nonlinear Hardening Material," ASME J. Appl. Mech., 53, 395.

1986 Watanabe, O. and Atluri, S.N., "Constitutive Modeling of Cyclic Plasticity and Creep Using an Internal Time Concept," Int. J. Plasticity, 2, 107.

1987 McDowell, D.L., “An Evaluation of Recent Developments in Hardening and Flow Rules for RateIndependent Nonproportional Cyclic Plasticity," ASME J. Appl. Mech. 54, 323.

1988 Khan, A. and Wang, X., "On Non-proportional Infinitesimal Plastic Deformation After Finite Plastic Prestraining and Partial Unloading," J. Mech. Phys. Solids, 36, 519.

1989a Inoue, T., Ohno, N., Suzuki, A. and Igari, T., "Evaluation of Inelastic Constitutive Models under Plasticity-Creep Interaction for $2 \frac{1}{4} \mathrm{Cr}-1 \mathrm{Mo}$ Steel at $600^{\circ} \mathrm{C}$," Nuclear Engng Design, 114, 295.

1989 b Inoue, T., Igari, T., Okazaki, M. and Sakane, M., "Fatigue-Creep Life Prediction Of $2 \frac{1}{4}$ Cr-1Mo Steel by Inelastic Analysis," Nuclear Engng Design, 114, 311.

1990 Lemaitre, J. and Chaboche, J., Mechanics of Solid Materials. Cambridge University Press, Cambridge.

1991a Inoue, T., Yoshida, F., Ohno, N., Kawai, M. and Niitsu, Y., "Evaluation of Inelastic Constitutive Models Under Plasticity-Creep Interaction In Multiaxial Stress State," Nuclear Engng Design, 126, 1.

1991 l Inoue, T., Okazaki, M., Igari, T., Sakane, M. and Kishi, S., "Evaluation of Fatigue-Creep Life Prediction Methods in Multiaxial Stress State," Nuclear Engng Design, 126, 13.

1993 Khan, A.S. and Wang, X., "An Experimental Study on Subsequent Yield Surface After Finite Shear Prestraining," Int. J. Plasticity, 9, 889.

1995 Khan, A.S. and Huang, S., Continuum Theory of Plasticity. Wiley, New York 